\title{
Midline (central) fluid percussion model of traumatic brain injury in pediatric and adolescent rats
}

\author{
Rachel K. Rowe, PhD, ${ }^{1-3}$ Jordan L. Harrison, PhD, $, 2,2,4$ Timothy W. Ellis, MBS, ${ }^{5}$ \\ P. David Adelson, MD, ${ }^{1,2}$ and Jonathan Lifshitz, $\mathrm{PhD}^{1-4}$ \\ 'Barrow Neurological Institute at Phoenix Children's Hospital; ' ${ }^{2}$ epartment of Child Health, University of Arizona College of \\ Medicine, Phoenix; ${ }^{3 P h o e n i x ~ V e t e r a n ~ A f f a i r s ~ H e a l t h c a r e ~ S y s t e m, ~ P h o e n i x ; ~}{ }^{4}$ Interdisciplinary Graduate Program in Neuroscience, \\ Arizona State University, Tempe; and 5Midwestern University, School of Osteopathic Medicine, Glendale, Arizona
}

OBJECTIVE Experimental traumatic brain injury (TBI) models hold significant validity to the human condition, with each model replicating a subset of clinical features and symptoms. TBI is the leading cause of mortality and morbidity in children and teenagers; thus, it is critical to develop preclinical models of these ages to test emerging treatments. Midline fluid percussion injury (FPI) might best represent mild and diffuse clinical brain injury because of the acute behavioral deficits, the late onset of behavioral morbidities, and the absence of gross histopathology. In this study, the authors sought to adapt a midline FPI to postnatal day (PND) 17 and 35 rats. The authors hypothesized that scaling the craniectomy size based on skull dimensions would result in a reproducible injury comparable to the standard midline FPI in adult rats.

METHODS PND17 and PND35 rat skulls were measured, and trephines were scaled based on skull size. Custom trephines were made. Rats arrived on PND10 and were randomly assigned to one of 3 cohorts: PND17, PND35, and 2 months old. Rats were subjected to midline FPI, and the acute injury was characterized. The right reflex was recorded, injury-induced apnea was measured, injury-induced seizure was noted, and the brains were immediately examined for hematoma.

RESULTS The authors' hypothesis was supported; scaling the trephines based on skull size led to a reproducible injury in the PND17 and PND35 rats that was comparable to the injury in a standard 2-month-old adult rat. The midline FPI suppressed the righting reflex in both the PND17 and PND35 rats. The injury induced apnea in PND17 rats that lasted significantly longer than that in PND35 and 2-month-old rats. The injury also induced seizures in 73\% of PND17 rats compared with $9 \%$ of PND35 rats and 0\% of 2-month-old rats. There was also a significant relationship between the righting reflex time and presence of seizure. Both PND17 and PND35 rats had visible hematomas with an intact dura, indicative of diffuse injury comparable to the injury observed in 2-month-old rats.

CONCLUSIONS With these procedures, it becomes possible to generate brain-injured juvenile rats (pediatric [PND17] and adolescent [PND35]) for studies of injury-induced pathophysiology and behavioral deficits, for which rational therapeutic interventions can be implemented.

https://thejns.org/doi/abs/10.3171/2018.1.PEDS17449

KEYWORDS traumatic brain injury; TBl; fluid percussion; pediatric; adolescent; aging; development; trauma

$\mathrm{T}$ Traumatic brain injury (TBI) is the leading cause of mortality and morbidity in children and teenagers. ${ }^{21} \mathrm{TBI}$ is more than a singular event, such that a disease state ensues, with morbidities developing and persisting over an extended timeframe after the initial insult. These chronic consequences are particularly profound for pediatric patients who have sustained a TBI, where de- cades of decreased quality of life can ensue. ${ }^{4}$ The longterm consequences of diffuse TBI can impair emotional, cognitive, and sensory function; however, despite these adverse outcomes after pediatric TBI, there is a paucity of basic science investigation into the mechanisms underlying the associated pathology and morbidity. ${ }^{1}$ The complex neurovascular responses after TBI require investigations

ABBREVIATIONS $\mathrm{FPI}=$ fluid percussion injury; $\mathrm{PND}=$ postnatal day; $\mathrm{TBI}=$ traumatic brain injury .

SUBMITTED August 10, 2017. ACCEPTED January 23, 2018.

INCLUDE WHEN CITING Published online April 20, 2018; DOI: 10.3171/2018.1.PEDS17449. 
that involve the immune, circulatory, and central nervous systems of live animals beyond the scope of in vitro and in silico modeling. A wide range of well-accepted animal models is available for neurotrauma investigations, which primarily differ in terms of the application of mechanical forces to the central nervous system. As clinical TBI is heterogeneous, each model addresses specific aspects of clinical pathophysiology. Together, the experimental brain injury literature applies injuries of mild to moderate severity in adult animals. Yet, this nomenclature translates into concussion or complicated concussion in the patient population. The approaches to induce brain injury and examine pathophysiology may be shared between adult and pediatric populations. Here, we outline the procedures to adapt this knowledge to the pediatric and adolescent rats.

Fluid percussion injury (FPI) is among the most well characterized and commonly used of all preclinical TBI models. ${ }^{14,23,24,39,41}$ FPI originally involved production of a midline injury and was modified shortly thereafter to the lateral injury site in adult rats. ${ }^{26}$ Midline FPI allows for the study of experimental TBI in a model that is reproducible, clinically relevant, and scalable between species and injury severities. Brain injury is induced by a rapid (approximately $20 \mathrm{msec}$ ) fluid pulse through a craniectomy onto the intact dura and creates an elastic decompression of the brain. ${ }^{8,40}$ The mechanical forces disrupt cell membranes, blood vessels, and neuronal processes without overt cell death. This model can be used to evaluate mechanisms of pathophysiology and behavioral impairments, and test therapeutic interventions that promote recovery of function.

While lateral FPI has previously been adapted to juvenile rats and is well established in the literature, $5,12,15,16,31$ midline FPI has yet to be adapted to juvenile rats. Lateral and midline FPIs use the same device and similar preparation surgeries, but key differences in the application and outcome provide unique value in each. We have adapted midline FPI for 2 reasons: 1) the absence of cortical tissue loss, cavitation, and overt cell death; and 2) no modification to the existing FPI device is needed. The purpose here is to provide a guide for adapting midline FPI to the juvenile rat.

The adaptations described in the current paper include the following 3 developmental time points that were se- lected after an extensive literature search of puberty and sexual maturation in rats: $:^{10,38,42,43}$ postnatal day (PND) 17, PND35, and 2 months. PND17 was chosen as a time point before the onset of puberty in rats and represents a pediatric age $(<3$ years old $)$ in humans. ${ }^{28,38}$ PND35 was chosen as a time point in the rat during puberty and represents the adolescent population (approximately 10-14 years old) in humans, and 2 months of age was chosen to represent adulthood after full sexual maturation (Fig. 1). Both PND17 and PND35 rats have been used in biomedical research to model children and young adults, including experimental brain injury research. 2,7,13,29,30,36

\section{Methods \\ Adaptation of Midline FPI to PND17 and PND35 Rats}

Fluid percussion brain injury has been successfully performed in various species, including cats, rabbits, pigs, rats, and mice. The adaptation of fluid percussion to rats ${ }^{9,25,26}$ was followed by its implementation in mice. ${ }^{6}$ The procedures outlined in this paper focus on PND17 (approximately 25-40 g) and PND35 (approximately 110-160 g) Sprague-Dawley rats. The following methods have been adapted from previously published studies using midline FPI in the mouse and adult rat. These methods highlight adaptations from our previously published methods. . $^{11,22,23,32,35}$

Adaptations to previously published surgical equipment include 3.0- and 4.0-mm-diameter custom trephines (Machine Shop, Arizona State University; Fig. 2). We designed and manufactured a 3-mm trephine for the PND17 rats and a 4.0-mm trephine for PND35 rats to accommodate the size difference of their skull compared with adult rats in which a $4.7-\mathrm{mm}$ trephine was used.

Preliminary studies were undertaken prior to the current study to identify a death curve for the PND17 and PND35 rats, as previously described. ${ }^{22}$ The death curve establishes the maximum atmospheric pressure that can be used to induce an FPI without mortality exceeding $20 \%$. For a moderate injury severity using the midline FPI model, mortality of $20 \%$ is expected within the acute posttraumatic period (15 minutes), due to respiratory failure and pulmonary edema. ${ }^{22}$ This expected mortality along with the righting reflex, an acute neurological evaluation
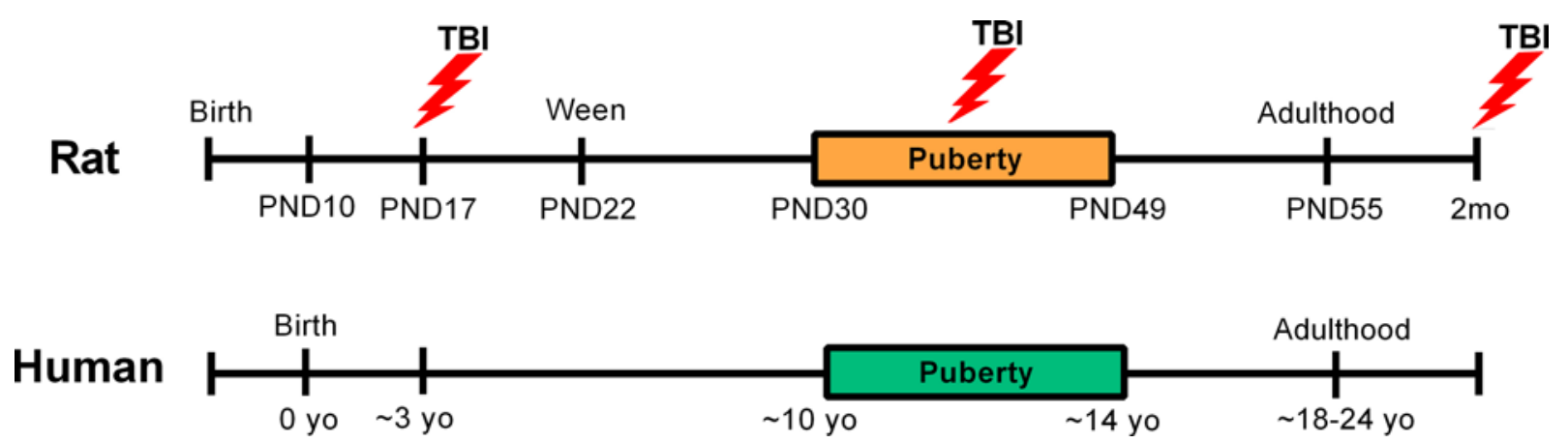

FIG. 1. Experimental timeline and comparison of developmental stages in rats and humans. The rats were subjected to TBI at PND17 before the onset of puberty, at PND35 during puberty, or at 2 months, which is after full sexual maturation and onset of adulthood in the rat. Puberty in humans occurs with an average age range of 10-14 years old (yo). Full sexual maturation and adulthood in humans occurs at approximately 18-24 years of age. 


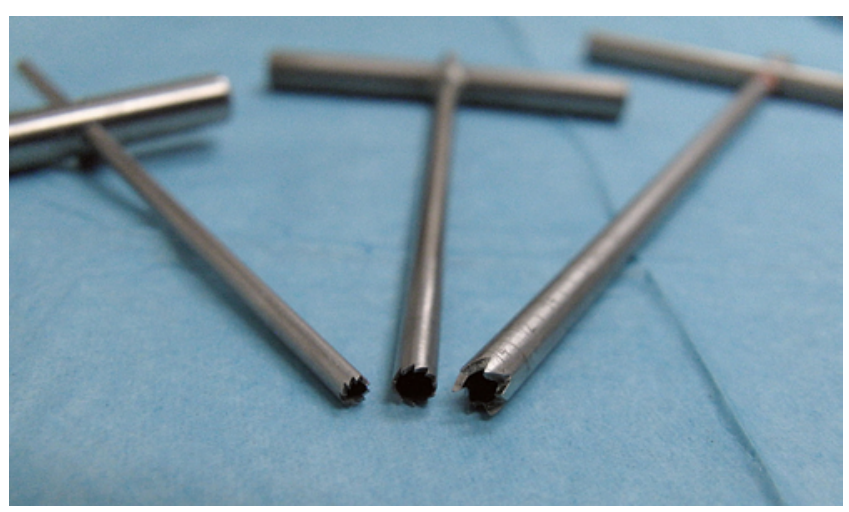

FIG. 2. Trephines adapted for the different stages of rat development. Custom-designed and machined 3.0- and 4.0-mm-diameter trephines (left and center, respectively) are displayed with the 4.7-mm trephine used in adult rats (right).

of injury severity, was used to establish the atmospheric pressure to induce the FPI. The hammer angle of the injury device was decreased for the younger rats to reduce the atmospheric pressure of the fluid pressure pulse (Table 1). For the current study, we sought to achieve a righting reflex time greater than 5 minutes and a mortality of less than $20 \%$. Mortality is a combination of injury pressure and surgical skill.

\section{Study Design}

The following experiments used one cohort of rats. All pups $(n=52)$ were shipped on PND10 with the dam in a single shipment and assigned to treatment groups on arrival. Groups were prepared for brain injury at PND17 ( $\mathrm{n}=$ 11 injury, $\mathrm{n}=6$ sham), PND35 ( $\mathrm{n}=12$ injury, $\mathrm{n}=6$ sham), or 2 months ( $n=11$ injury, $n=6$ sham; Fig. 1$)$. The rats were subjected to midline FPI, as previously described. ${ }^{11,22}$ All animal studies were conducted in accordance with the guidelines established by the internal Institutional Animal Care and Use Committee at the University of Arizona and the National Institutes of Health Guide for the Care and Use of Laboratory Animals. The study is reported following the ARRIVE (Animal Research: Reporting In Vivo Experiments) guidelines. ${ }^{19}$ Randomization of animals was achieved by assigning animals to treatment (age at injury) groups before the initiation of the study to ensure equal distribution across groups. A power analysis was performed to calculate group sizes to enable statistically robust detection of injury-induced deficits while minimizing the number of animals. This calculation was based on preliminary data from our group. Data collection was stopped at predetermined final end points based on days postinjury for each animal. All data analyses were conducted by investigators blinded to the treatment groups. Data sets were screened using the extreme Studentized deviate method for significant outliers. No significant outliers were identified. The rats were acclimated to their environment after shipment for at least 1 week prior to any experiments. After injury or sham surgery, the rats were evaluated daily during postoperative care by a physical examination and each animal's condition was documented. PND17 rats were returned to the dam following brain injury. One rat (PND35) died af-
TABLE 1. Summary of adaptations to the FPD and mortality rates*

\begin{tabular}{lccc}
\hline \multirow{2}{*}{ Variable } & \multicolumn{3}{c}{ Rat Group } \\
\cline { 2 - 4 } & PND17 & PND35 & 2-Mo-Old \\
\hline Angle (degrees) & $11.0-12.5$ & $14.5-15.5$ & 16.0 \\
\hline Pressure (atm) & $1.3-1.5$ & $1.8-2.0$ & $1.9-2.2$ \\
\hline Mortality & $0 / 11$ & $1 / 12$ & $0 / 11$ \\
\hline
\end{tabular}

* To adapt the midline FPI to juveniles, the hammer angle of the injury device was lowered to reduce the pressure of the pressure pulse. Lower pressure was used on juveniles to prevent injury-induced mortality and maintain righting reflex times. Sham animals were not subjected to injury and are not included in this table.

ter injury and was excluded from all analyses. The shaminjured rats were used as a control for the craniectomy and anesthesia. No sham rat had a righting reflex longer than 10 seconds, apnea, seizure, or presence of a hematoma. Shams are not included in the statistical analyses.

\section{Midline FPI}

The rat was anesthetized using 5\% isoflurane in $100 \%$ oxygen for 5 minutes, and its head was placed in a stereotactic frame, with continuously delivered isoflurane at $2.5 \%$ via a nosecone. While the rats were anesthetized, their body temperature was maintained using a Deltaphase isothermal heating pad (Braintree Scientific Inc.). A midline sagittal incision was made, extending from between the eyes to the base of the skull, just past the ears (Figs. 3B and 4B).

\section{Cranial Surgery for Hub Placement-PND17}

For PND17 rats, a disc was cut from weed trimmer line and placed on the skull midway between the bregma and lambda on the sagittal suture to guide the trephine (Fig. 3C). A trephine (3-mm outer diameter) was used for the craniectomy centered on the sagittal suture between the bregma and lambda. Under a microscope, the bone was removed without disrupting the underlying dura, and the craniectomy site was irrigated with saline to remove any blood (Fig. 3D).

\section{Cranial Surgery for Hub Placement-PND35}

The location on the skull for the craniectomy was marked (midway between the bregma and lambda, over the sagittal suture), and a pilot hole was made using a Dremel tool (engraving cutter 106; Fig. 4C). A custom trephine (4.0-mm outer diameter) was used for the craniectomy by anchoring the centering pin in the pilot hole at the craniectomy center. The bone was removed without disruption of the underlying dura, and the craniectomy site was irrigated with saline (Fig. 4D).

\section{Injury Hub Placement}

After removal of the bone from the craniectomy site, a hub was constructed from a 22-gauge, 1.5 -inch needle. The female Luer-Loc hub was cut from the needle with an outer diameter of $3.0 \mathrm{~mm}$ for PND17 rats and $4.0 \mathrm{~mm}$ for PND35 rats. The injury hub was placed outside the crani- 

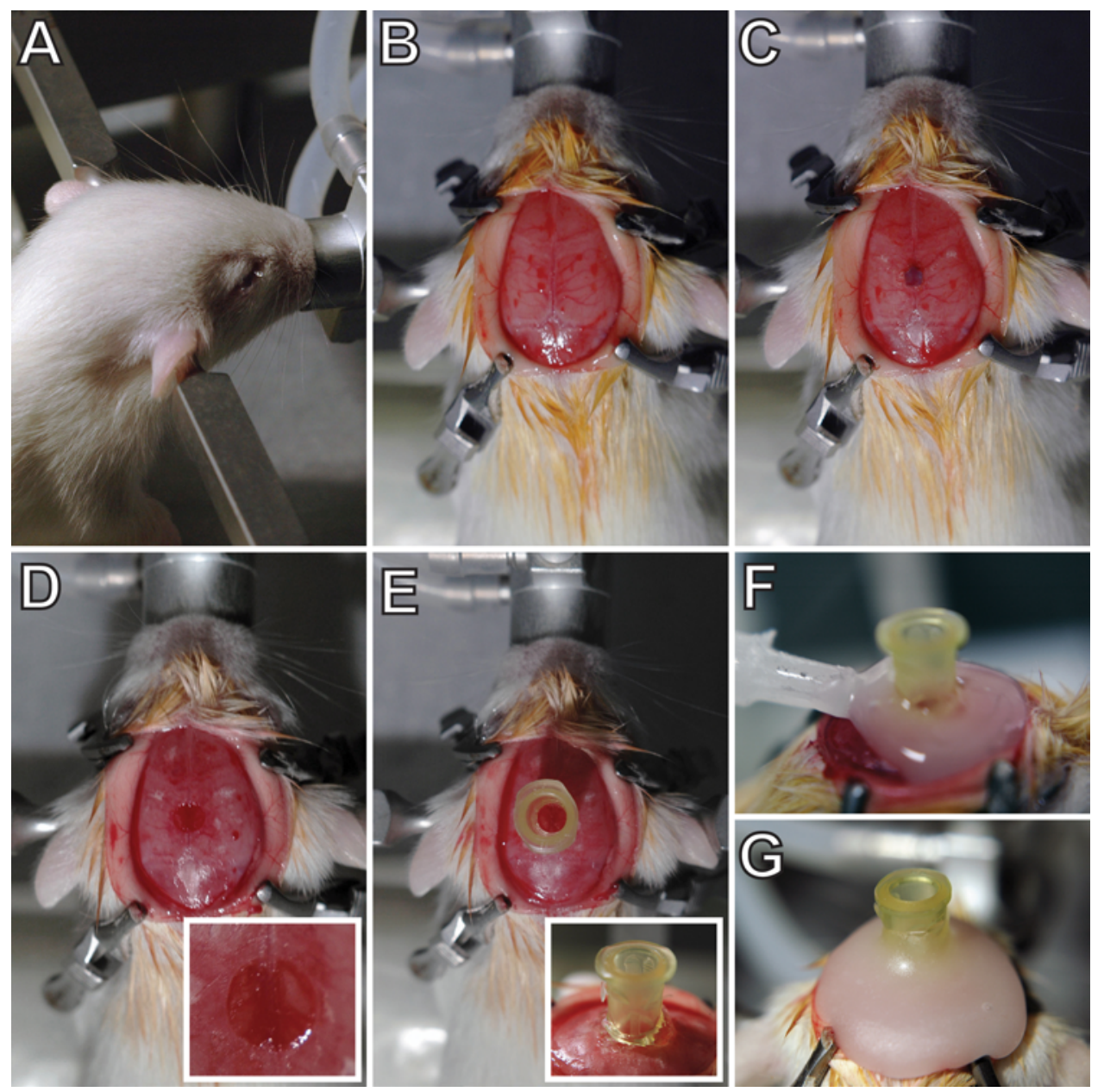

FIG. 3. Cranial surgery for hub placement in the pediatric (PND17) rat. A: The head of the rat is swabbed with alcohol and Betadine and secured in a stereotactic frame with a continuous flow of inhaled isoflurane via a nosecone. B: A midline scalp incision is made to expose the skull, and the overlying fascia is removed. C: Vetbond (3M) tissue adhesive is applied to secure a thin (translucent) cross-section of weed trimmer line directly on the midline between the bregma and lambda sutures. D: Using the weed trimmer line as an anchor, a 3.0-mm-diameter trephine is used to perform a craniectomy to expose the underlying dura. E: Small drops of cyanoacrylate gel are placed on the outside of the constructed injury hub, and the hub is centered atop the craniectomy. F and G: After the cyanoacrylate gel dries, the injury hub is covered in methyl methacrylate cement, and the injury hub is filled with saline.

ectomy and secured with cyanoacrylate gel applied just above the cut end to create a seal. Careful attention was taken to ensure that the injury hub was secured perpendicular to the dural surface. When the cyanoacrylate gel was dry, the injury hub was covered in methyl methacrylate cement (Figs. 3F and $\mathrm{G}$ and $4 \mathrm{~F}$ ).

\section{Injury Induction}

For injury induction, the rats were reanesthetized with $5 \%$ isoflurane delivered for 3 minutes after a 60 -minute recovery period from surgery. The inside of the injury hub was inspected to ensure that the dura was intact with no debris. The hub was then filled with normal saline (Fig. $5 \mathrm{~A}$ and $\mathrm{B}$ ), and the female Luer-Loc injury hub on the rat was connected to the male Luer-Loc fitting on the injury device (Fig. 5A-D). PND17 rats were attached to the device using an extension tube and held lying on their right side (Fig. 5C). PND35 rats were attached directly to the device and held laterally on their right side (Fig. 5D). The rats were checked for a positive toe-pinch response, after which the injury was administered by releasing the pendulum to strike the plunger of the fluid-filled cylinder. Sham-injured rats underwent the same procedure, except the pendulum was not released. After pendulum release or sham injury, the injury hub was removed, and the brain was inspected for uniform herniation and integrity of the dura. The dura was intact in all rats; none were excluded as technical or surgical failures. The rats were monitored for the presence of seizures, and righting reflex times were recorded for the injured rats as indicators of injury severity. ${ }^{18}$ The righting reflex time is the total time from the initial impact until the rat spontaneously rights itself from a supine position. After spontaneous righting, the incision was cleaned using saline and sutured closed. All sham-injured rats recovered a righting reflex immediately after removal from the device (within 10 seconds). After 

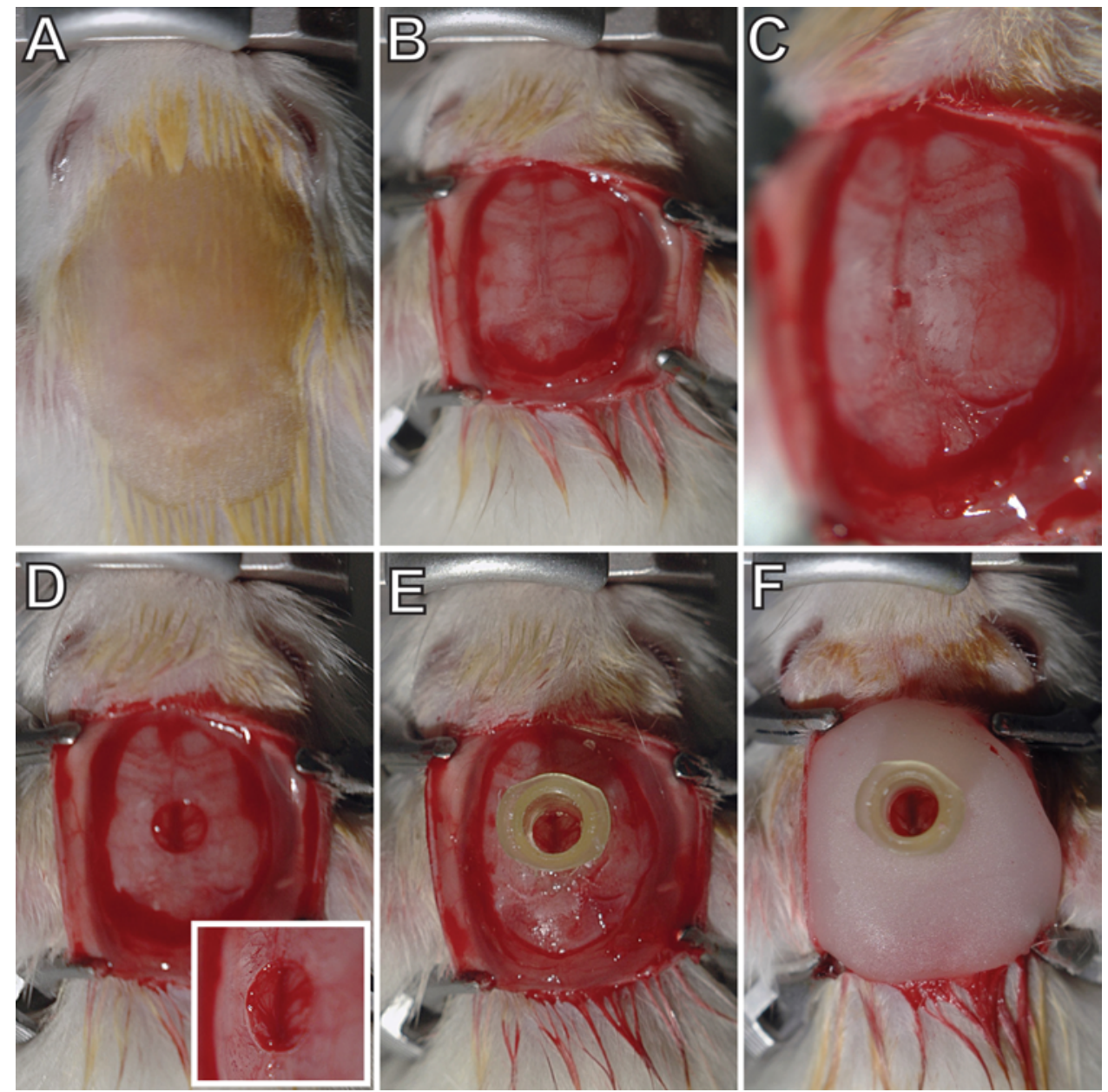

FIG. 4. Cranial surgery for hub placement in the adolescent (PND35) rat. A: The head of the rat is shaved, swabbed with alcohol and Betadine, and secured in a stereotactic frame with a continuous flow of inhaled isoflurane via a nosecone. B: A midline scalp incision is made to expose the skull, and the overlying fascia is removed. C: A Dremel tool with a burr bit is used to make a pilot hole to anchor the trephine via a centering pin. D: A 4.0-mm-diameter trephine is used to perform the craniectomy to expose the underlying dura. E: Small drops of cyanoacrylate gel are placed on the outside of the constructed injury hub, and the hub is centered atop the craniectomy. F: After the cyanoacrylate gel dries, the injury hub is covered in methyl methacrylate cement, and the injury hub is filled with saline.

spontaneously righting, the rats were placed in a heated recovery cage and monitored until they were ambulatory (approximately $5-15$ additional minutes) before being returned to their home cage. Special care was taken to clean PND17 rats using sterile saline to prevent rejection from the dam. Adequate measures were taken to minimize pain or discomfort. ${ }^{34}$

\section{Tissue Collection}

The rats were administered an overdose of intraperitoneal sodium pentobarbital and transcardially perfused with $4 \%$ paraformaldehyde after a phosphate-buffered saline flush. The brains were examined for gross pathology and placed in $4 \%$ paraformaldehyde overnight. They were then immersed in serial dilutions (15\% and 30\%) of sucrose and frozen at $-40^{\circ} \mathrm{C}$, then stored at $-80^{\circ} \mathrm{C}$.

\section{Statistical Analysis}

Data are shown as the mean \pm SEM and were analyzed using GraphPad Prism 6, with statistical significance assigned at $\mathrm{p}<0.05$. Differences in righting reflex times and apnea duration were measured using 1-way ANOVA. All significant data were further analyzed using Tukey's multiple comparison test. To determine if the righting reflex time was influenced by the presence of a seizure, a linear regression model was implemented in R 3.3.0 (R Core Team). Sham values were not included in the statistical analyses.

\section{Results}

\section{Midline FPI Suppressed Righting Reflex Response in PND17 and PND35 Rats}

We have previously reported suppression of the righting reflex response in adult rats ${ }^{18}$ and mice ${ }^{17,33}$ after midline FPI as an injury-induced deficit and an indicator of injury severity. Diffuse brain injury suppressed the righting reflex response in PND17 and PND35 rats. All uninjured shams recovered a righting reflex within 20 seconds, as is 

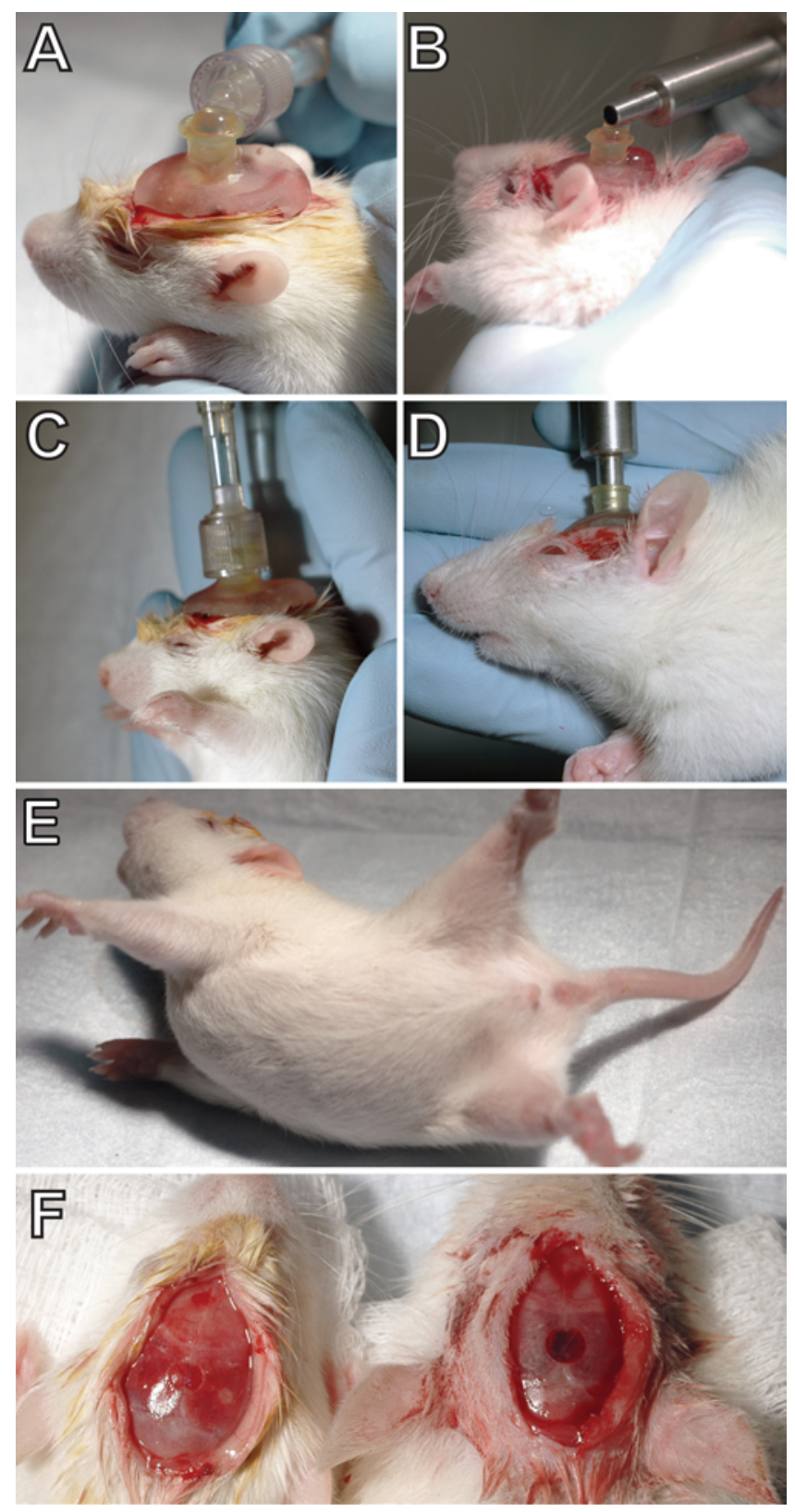

FIG. 5. Midline FPI in the PND17 and PND35 rats. A and B: The injury hub is filled with sterile saline and connected to the male fitting of the injury device. PND17 rats are connected to the device via an extension tube (A), and PND35 rats are connected directly to the device (B). C and D: The PND17 (C) or PND35 (D) rat is positioned and held parallel to the ground on its right side. The pendulum is released once a positive toe-pinch response is achieved. E: The rats are placed supine on a heating pad and monitored until restoration of the righting reflex. Of note, in this adaptation we observed a high incidence of acute postinjury seizure in PND17 rats. F: The condition/appearance of the surgical site and brain tissue beneath the injury site is observed for herniation and hematoma (left, uninjured sham; right, brain-injured rat).

expected after short-duration, inhaled anesthesia. A 1-way ANOVA indicated an overall effect of righting reflex time between groups $(\mathrm{F}(2,30)=5.499, \mathrm{p}=0.0092$; Fig. 6A). Further analysis using Tukey's multiple comparisons test indicated that PND17 rats had a significantly shorter right- ing reflex time than that for PND35 rats. However, there was no significant difference between PND17 and PND35 rats and the 2-month-old young adult rats.

\section{Midline FPI Induced Apnea in PND17 Rats}

The rats were not intubated after injury, and there was no manual intervention to start respiration. All rats recovered spontaneous respiration after midline FPI. PND17 and PND35 rats, but not 2-month-old adult rats, experienced a cessation of breathing as denoted by the absence of chest movement associated with breathing. There was a significant overall effect of apnea duration $(F(2,30)=$ $10.60, p=0.0003$; Fig. 6B). Further analyses indicated that PND17 rats had significantly longer durations of apnea than did the PND35 and 2-month-old adult rats.

\section{Midline FPI Induced Seizures in PND17 Rats}

Following midline FPI, 73\% (8/11) of PND17 rats experienced seizures compared with 9\% (1/11) of PND35 rats and $0 \%(0 / 11)$ of adults (Fig. 6C). Seizures were defined as one or more of the following observed characteristic movements: unnatural body contortions, vigorous tail whipping or spinning, or spontaneous overt muscle contraction/relaxation (Fig. 6E). The observed seizures mimicked and resembled those seen in clinic and were most similar to those described as tonic-clonic. ${ }^{27}$ Linear regression indicated a significant relationship between the righting reflex and the presence of seizure, in which the righting reflex time decreased by 92.26 seconds on average $(95 \% \mathrm{CI}-142.392$ to $-42.127 ; \mathrm{t}=-3.753, \mathrm{p}=0.000721$; Fig. 6D), if the animal had an injury-induced seizure $\left(\mathrm{r}^{2}=\right.$ $0.29, \mathrm{f}=14.09, \mathrm{df}=31$ ).

\section{Midline FPI Resulted in Hematoma in Juvenile Rats}

Immediately after induction of injury, a craniotomy was performed and the brain was examined for herniation and hematoma, which occurred after recovery of the righting reflex and prior to closing the incision with sutures. As seen in the adult midline FPI model, both PND17 and PND35 rats had significant hematomas immediately after the injury (Fig. 5F). The presence of hematoma with an intact dura is indicative of the diffuse injury caused by the midline FPI and further confirms a comparable injury between adult and juvenile rats. Uninjured rats have an intact dura and no hematoma (Figs. 3D and 4D). None of the animals exhibited herniation of cortical tissue through the craniectomy, a characteristic of a compromised dura.

\section{Discussion}

The adaptation of the FPI model for juvenile animals addresses an increasing need for techniques to study TBI throughout the lifespan, including development. While other models of experimental brain injury, including lateral FPI, have been adapted for juvenile rats, here, we provide the first protocol to adapt the midline FPI model for PND17 and PND35 rats. In the adaptation of FPI described here, the trephine diameter is scaled for different-aged rats to account for skull size. To scale the trephine, the distance was measured between the lambda and bregma at all 3 ages, and the trephines were manufactured with di- 

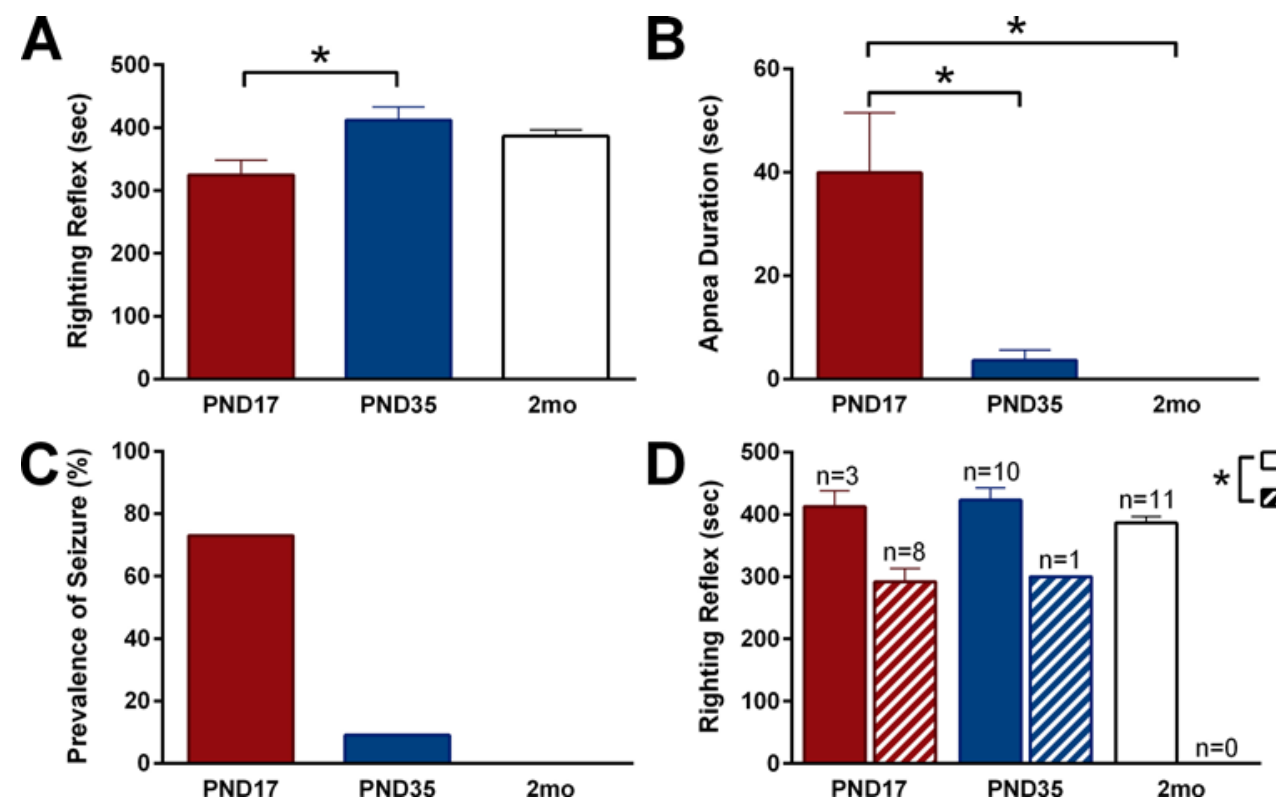

FIG. 6. Acute physiological characterization of the adapted midline FPI. A: Midline FPI suppressed the righting reflex response in all rats. PND17 rats had a significantly shorter righting reflex time than did PND35 rats. However, there was no difference between PND17 $(n=11)$ and PND35 $(n=11)$ rats and the 2-month-old adult rats $(n=11)$. B: Midline FPI induced apnea in juvenile but not adult rats. PND17 rats had significantly longer apnea durations than those for PND35 and 2-month-old adult rats. C: Midline FPI induced seizures in PND17 but not adult rats; $73 \%$ of PND17 rats experienced seizures compared with $9 \%$ of PND35 rats and $0 \%$ of adults. D: There was a significant relationship between righting reflex times and the presence of seizure in which righting reflex times decreased by 92.26 seconds on average if the animal had a seizure. Data are presented as the mean $\pm S E M$; ${ }^{*} p<0.05$. Sham values were not included in the analyses.

ameters of approximately $50 \%$ of this distance. This detail enhances the scalability of the injury severity across ages. Using a 4.7-mm trephine, commonly used on adult rats, to produce the craniectomy for a PND35 rat exposes a larger portion of the brain to the fluid pulse, and produces results that are inconsistent with the injury administered to the PND17 and adult rats. Scaling the trephine sizes based on skull size is an essential consideration for all experimental brain injury models.

With the recognition of an epidemic number of cases of TBI among the pediatric population, there is a need for translational research to investigate the lifelong burden of TBI in children. However, research in the preclinical field specific to pediatric TBI models is limited. In total, there are currently 6 models of experimental TBI in the developing rat: weight drop, lateral fluid percussion, impact acceleration, controlled cortical impact, mechanical shaking, and closed-head cortical impact. For a comprehensive review of current preclinical models for pediatric TBI see Kochanek et al. ${ }^{20}$ Each experimental model has value in addressing specific hypotheses. For all preclinical pediatric research, it is imperative to follow the animal as it ages with the injury and be able to compare functional outcome measures across development. It is also important to be able to compare the injury response and the secondary injury burden in a developing animal to that in an adult animal sustaining a similar injury with the same experimental model. For this reason, we found it necessary to adapt the midline FPI for developing rats. The midline FPI is a well-characterized and prevalently used experimental model in adult rats. Adapting this model for developing rats provides a research tool to expand translational pediatric research and provides research laboratories currently using midline FPI with a tool to compare injury across multiple ages.

In the current study, we found that a midline FPI produces physiological responses in the PND17 rats that differ from those of the PND35 and adult rats. We observed shorter righting reflex times in the PND17 rats that were not statistically different from those in adult rats, but significantly shorter than the righting reflex times of the PND35 rats. PND17 rats also experienced significantly longer durations of injury-induced apnea, and a higher proportion experienced injury-induced seizures. Injury-induced seizures occurred in 73\% of PND17 rats compared with 9\% of PND35 rats and $0 \%$ of adult rats. This is consistent with clinical data published by our group and collaborators in which early posttraumatic seizures were a common complication in 0- to 3-year-old children after TBI. ${ }^{3}$ During a seizure, the sudden abnormal electrical disturbance in the brain may extend to decrease the righting reflex times. ${ }^{37}$ PND17 rats that experienced a seizure regained the righting reflex almost immediately following the conclusion of the seizure. Statistical analysis confirmed that the presence of seizures contributed to shorter righting reflex times. Whether a shorter righting reflex time with a seizure is comparable to a longer righting reflex time remains to be determined. To date, the seizure etiology in PND17 immediately following midline FPI is unknown.

We have previously used righting reflex times as an indicator of injury severity after midline FPI in rodents; ${ }^{17,18}$ however, in the current study, the presence of seizures po- 
tentially confounds this injury severity marker. After the injury, prior to closing the incision, hematomas were visible under the skull of both PND17 and PND35 rats, which is consistent with visible hematomas in adult rodents subjected to midline FPI. It is necessary for future work with this injury model to be aimed at correlating injury severity to pathological outcome measures, including hematoma, blood-brain barrier disruption, and inflammation. Furthermore, injury-induced seizures may affect chronic pathology and behavioral outcomes in PND17 rats, and it is necessary for future work to compare histological findings in PND17 rats in the presence and absence of seizures. However, the diffuse nature of midline fluid percussion occurs in the absence of cavitation or overt contusion, leaving sensitive neuropathological markers (e.g., silver stain) to identify injury severity. Also, the biomechanics of the pressure pulse on the brain within the skull needs to be compared across age groups. It is possible that the thin skull of the PND17 rats promotes extrusion of the brain through the foramen magnum, such that forces impinge on the brainstem and induce apnea in response to the pressure pulse. Our ongoing research focuses on the histopathological and behavioral outcome measures in the PND17 pediatric and PND35 adolescent rats, which will positively impact the field of pediatric TBI.

\section{Conclusions}

We have outlined the successful adaption of the midline FPI model for juvenile rats. There is an increasing need for techniques to study TBI throughout the lifespan, including during development. While other models of experimental brain injury, including lateral FPI, have been adapted for juvenile rats, in this paper we have provided the first protocol to adapt the midline FPI model for PND17 and PND35 rats. For the adaptation of the FPI presented here, we scaled the trephine diameter for rats of different ages to account for skull size. This is the first study of its kind to scale craniectomy size across developing ages of rats to provide a valid injury model for pediatric brain injury.

\section{Acknowledgments}

This work was supported in part by Phoenix Children's Hospital Mission Support Funds. Dr. Rowe is funded by a Bisgrove Scholar Award from Science Foundation Arizona. Dr. Harrison was partially supported by the Diane and Bruce Halle Foundation and NIH grant no. F31 NS09092. We thank Dr. Sean Murphy for contribution to the statistical analyses of data.

\section{References}

1. Acerini CL, Tasker RC, Bellone S, Bona G, Thompson CJ, Savage MO: Hypopituitarism in childhood and adolescence following traumatic brain injury: the case for prospective endocrine investigation. Eur J Endocrinol 155:663-669, 2006

2. Adelson PD, Dixon CE, Kochanek PM: Long-term dysfunction following diffuse traumatic brain injury in the immature rat. J Neurotrauma 17:273-282, 2000

3. Arango JI, Deibert CP, Brown D, Bell M, Dvorchik I, Adelson PD: Posttraumatic seizures in children with severe traumatic brain injury. Childs Nerv Syst 28:1925-1929, 2012

4. Babikian T, Merkley T, Savage RC, Giza CC, Levin H: Chronic aspects of pediatric traumatic brain injury: review of the literature. J Neurotrauma 32:1849-1860, 2015
5. Babikian T, Prins ML, Cai Y, Barkhoudarian G, Hartonian I, Hovda DA, et al: Molecular and physiological responses to juvenile traumatic brain injury: focus on growth and metabolism. Dev Neurosci 32:431-441, 2010

6. Carbonell WS, Maris DO, McCall T, Grady MS: Adaptation of the fluid percussion injury model to the mouse. J Neurotrauma 15:217-229, 1998

7. Casella EM, Thomas TC, Vanino DL, Fellows-Mayle W, Lifshitz J, Card JP, et al: Traumatic brain injury alters long-term hippocampal neuron morphology in juvenile, but not immature, rats. Childs Nerv Syst 30:1333-1342, 2014

8. Dixon CE, Lighthall JW, Anderson TE: Physiologic, histopathologic, and cineradiographic characterization of a new fluid-percussion model of experimental brain injury in the rat. J Neurotrauma 5:91-104, 1988

9. Dixon CE, Lyeth BG, Povlishock JT, Findling RL, Hamm RJ, Marmarou A, et al: A fluid percussion model of experimental brain injury in the rat. J Neurosurg 67:110-119, 1987

10. Döhler KD, Wuttke W: Serum LH, FSH, prolactin and progesterone from birth to puberty in female and male rats. Endocrinology 94:1003-1008, 1974

11. Eakin K, Rowe RK, Lifshitz J: Modeling fluid percussion injury: relevance to human traumatic brain injury, in Kobeissy FH (ed): Brain Neurotrauma: Molecular, Neuropsychological, and Rehabilitation Aspects. Boca Raton: CRC Press, 2015

12. Giza CC, Griesbach GS, Hovda DA: Experience-dependent behavioral plasticity is disturbed following traumatic injury to the immature brain. Behav Brain Res 157:11-22, 2005

13. Greco T, Hovda DA, Prins ML: Adolescent TBI-induced hypopituitarism causes sexual dysfunction in adult male rats. Dev Neurobiol 75:193-202, 2015

14. Groat RA, Windle WF, Magoun HW: Functional and structural changes in the monkey's brain during and after concussion. J Neurosurg 2:26-35, 1945

15. Gurkoff GG, Giza CC, Hovda DA: Lateral fluid percussion injury in the developing rat causes an acute, mild behavioral dysfunction in the absence of significant cell death. Brain Res 1077:24-36, 2006

16. Gurkoff GG, Giza CC, Shin D, Auvin S, Sankar R, Hovda DA: Acute neuroprotection to pilocarpine-induced seizures is not sustained after traumatic brain injury in the developing rat. Neuroscience 164:862-876, 2009

17. Harrison JL, Rowe RK, Ellis TW, Yee NS, O'Hara BF, Adelson PD, et al: Resolvins AT-D1 and E1 differentially impact functional outcome, post-traumatic sleep, and microglial activation following diffuse brain injury in the mouse. Brain Behav Immun 47:131-140, 2015

18. Hosseini AH, Lifshitz J: Brain injury forces of moderate magnitude elicit the fencing response. Med Sci Sports Exerc 41:1687-1697, 2009

19. Kilkenny C, Browne WJ, Cuthill IC, Emerson M, Altman DG: Improving bioscience research reporting: the ARRIVE guidelines for reporting animal research. PLoS Biol 8:e1000412, 2010

20. Kochanek PM, Wallisch JS, Bayır H, Clark RSB: Pre-clinical models in pediatric traumatic brain injury-challenges and lessons learned. Childs Nerv Syst 33:1693-1701, 2017

21. Langlois JA, Rutland-Brown W, Wald MM: The epidemiology and impact of traumatic brain injury: a brief overview. $\mathbf{J}$ Head Trauma Rehabil 21:375-378, 2006

22. Lifshitz J: Fluid percussion injury model, in Chen J, Xu XM, Xu ZC, et al (eds): Animal Models of Acute Neurological Injuries. Totowa, NJ: Humana Press, 2009

23. Lifshitz J, Rowe RK, Griffiths DR, Evilsizor MN, Thomas TC, Adelson PD, et al: Clinical relevance of midline fluid percussion brain injury: acute deficits, chronic morbidities and the utility of biomarkers. Brain Inj 30:1293-1301, 2016

24. Lindgren S, Rinder L: Experimental studies in head injury. I. 
Some factors influencing results of model experiments. Biophysik 2:320-329, 1965

25. McIntosh TK, Noble L, Andrews B, Faden AI: Traumatic brain injury in the rat: characterization of a midline fluidpercussion model. Cent Nerv Syst Trauma 4:119-134, 1987

26. McIntosh TK, Vink R, Noble L, Yamakami I, Fernyak S, Soares H, et al: Traumatic brain injury in the rat: characterization of a lateral fluid-percussion model. Neuroscience 28:233-244, 1989

27. Morrell MJ: Differential diagnosis of seizures. Neurol Clin 11:737-754, 1993

28. Mu D, Jiang X, Sheldon RA, Fox CK, Hamrick SE, Vexler ZS, et al: Regulation of hypoxia-inducible factor $1 \alpha$ and induction of vascular endothelial growth factor in a rat neonatal stroke model. Neurobiol Dis 14:524-534, 2003

29. Prins ML, Fujima LS, Hovda DA: Age-dependent reduction of cortical contusion volume by ketones after traumatic brain injury. J Neurosci Res 82:413-420, 2005

30. Prins ML, Hovda DA: The effects of age and ketogenic diet on local cerebral metabolic rates of glucose after controlled cortical impact injury in rats. J Neurotrauma 26:1083-1093, 2009

31. Prins ML, Lee SM, Cheng CL, Becker DP, Hovda DA: Fluid percussion brain injury in the developing and adult rat: a comparative study of mortality, morphology, intracranial pressure and mean arterial blood pressure. Brain Res Dev Brain Res 95:272-282, 1996

32. Rowe RK, Griffiths DR, Lifshitz J: Midline (central) fluid percussion model of traumatic brain injury, in Kobeissy FH, Dixon CE, Hayes RL, et al (eds): Injury Models of the Central Nervous System: Methods and Protocols. New York: Springer, 2016, pp 211-230

33. Rowe RK, Harrison JL, O'Hara BF, Lifshitz J: Recovery of neurological function despite immediate sleep disruption following diffuse brain injury in the mouse: clinical relevance to medically untreated concussion. Sleep (Basel) 37:743-752, 2014

34. Rowe RK, Harrison JL, Thomas TC, Pauly JR, Adelson PD, Lifshitz J: Using anesthetics and analgesics in experimental traumatic brain injury. Lab Anim (NY) 42:286-291, 2013

35. Rowe RK, Striz M, Bachstetter AD, Van Eldik LJ, Donohue $\mathrm{KD}$, O'Hara BF, et al: Diffuse brain injury induces acute post-traumatic sleep. PLoS One 8:e82507, 2013

36. Rowe RK, Ziebell JM, Harrison JL, Law LM, Adelson PD, Lifshitz J: Aging with traumatic brain injury: effects of age at injury on behavioral outcome following diffuse brain injury in rats. Dev Neurosci 38:195-205, 2016

37. Schwartzkroin PA, Roper SN, Wenzel HJ: Cortical dysplasia and epilepsy: animal models. Adv Exp Med Biol 548:145174,2004

38. Sengupta P: The laboratory rat: relating its age with human's. Int J Prev Med 4:624-630, 2013
39. Sullivan HG, Martinez J, Becker DP, Miller JD, Griffith R, Wist AO: Fluid-percussion model of mechanical brain injury in the cat. J Neurosurg 45:521-534, 1976

40. Thibault LE, Meaney DF, Anderson BJ, Marmarou A: Biomechanical aspects of a fluid percussion model of brain injury. J Neurotrauma 9:311-322, 1992

41. Thompson HJ, Lifshitz J, Marklund N, Grady MS, Graham DI, Hovda DA, et al: Lateral fluid percussion brain injury: a 15-year review and evaluation. J Neurotrauma 22:42-75, 2005

42. Urbanski HF, Ojeda SR: The juvenile-peripubertal transition period in the female rat: establishment of a diurnal pattern of pulsatile luteinizing hormone secretion. Endocrinology 117:644-649, 1985

43. Zemunik T, Peruzovic M, Capkun V, Zekan L, Tomic S, Milkovic K: Reproductive ability of pubertal male and female rats. Braz J Med Biol Res 36:871-877, 2003

\section{Disclosures}

The authors report no conflict of interest concerning the materials or methods used in this study or the findings specified in this paper.

\section{Author Contributions}

Conception and design: Rowe, Ellis, Lifshitz. Acquisition of data: Rowe, Harrison, Ellis. Analysis and interpretation of data: Rowe, Harrison. Drafting the article: Rowe, Harrison. Critically revising the article: Rowe, Harrison, Adelson, Lifshitz. Reviewed submitted version of manuscript: all authors. Approved the final version of the manuscript on behalf of all authors: Rowe. Statistical analysis: Rowe. Study supervision: Rowe, Adelson, Lifshitz.

\section{Supplemental Information \\ Previous Presentations}

Portions of this work were presented in abstract/poster form at the 34th Annual National Neurotrauma Symposium, Lexington, Kentucky, June 26-29, 2016.

\section{Current Affiliations}

Dr. Harrison: Basic Medical Sciences, University of Arizona College of Medicine, Phoenix, AZ.

Mr. Ellis: Midwestern University, College of Dental Medicine, Glendale, AZ.

\section{Correspondence}

Rachel K. Rowe: Barrow Neurological Institute, Phoenix Children's Hospital, Phoenix, AZ.rkro222@email.arizona.edu. 